Pham Van Thu and Nguyen Dinh Duc*

\title{
Non-linear dynamic response and vibration of an imperfect three-phase laminated nanocomposite cylindrical panel resting on elastic foundations in thermal environments
}

DOI 10.1515/secm-2015-0467

Received November 11, 2015; accepted March 19, 2016; previously published online May 3, 2016

\begin{abstract}
This paper presents an analytical approach to investigate the non-linear dynamic response and vibration of an imperfect three-phase laminated nanocomposite cylindrical panel resting on elastic foundations in thermal environments. Based on the classical laminated shell theory and stress function, taking into account geometrical non-linearity, initial geometrical imperfection, Pasternak-type elastic foundation, and temperature, the governing equations of the three-phase laminated nanocomposite cylindrical panel are derived. The numerical results for the dynamic response and vibration of the polymer nanocomposite cylindrical panel are obtained by using the Runge-Kutta method. The influences of fibres and nanoparticles, different fibre angles, material and geometrical properties, imperfection, elastic foundations, and temperature on the non-linear dynamic response of the polymer nanocomposite cylindrical panel are discussed in detail.
\end{abstract}

Keywords: elastic foundations; non-linear dynamic; thermal environments; three-phase laminated nanocomposite cylindrical panel; vibration.

\section{Introduction}

Laminated composites have become indispensable in several modern-day applications, such as for use in highperformance structures in the fields of civil, marine, and aerospace engineering, among others. Therefore, the

*Corresponding author: Nguyen Dinh Duc, Vietnam National University, Hanoi, 144 Xuan Thuy, Cau Giay, Hanoi, Vietnam, Phone: +84-4-37547978, Fax: +84-4-37547724, e-mail: ducnd@vnu.edu.vn

Pham Van Thu: Nha Trang University, 44 Hon Ro, Nha Trang, Khanh Hoa, Vietnam static and dynamic stabilities of sandwich laminated composite structures have attracted the attention of many researchers around the world. Jin et al. [1] presented the use of a digital image correlation technique to investigate the thermal buckling of a circular laminated composite plate subjected to a uniform distribution of temperature load. Lei et al. [2] investigated the first-known vibration analysis of thin to moderately thick laminated functionally graded carbon nanotube reinforced composite rectangular plates. Rossol et al. [3] developed a meso-scale finite element model to study the effects of weave architecture on the strain and stress evolution in an eightharness satin $\mathrm{SiC} / \mathrm{SiCN}$ composite. Yatim et al. [4] studied the strength and behaviour of simple supported partially connected composite plate girders, while Garcia et al. [5] suggested a methodology for delamination assessment in free vibrating composite laminate plates. Aykul [6] carried out a residual stress analysis on symmetric and anti-symmetric cross-ply and angle-ply thermoplastic simple supported laminated plates for transverse loading. Based on isogeometric analysis and higher-order shear deformation theory, Loc et al. [7] researched the geometrically nonlinear analysis of laminated composite plates. Ansari et al. [8] proposed an efficient numerical method in the context of variational formulation and on the basis of the Rayleigh-Ritz technique to address the free vibration problem of laminated composite conical shells. Jun et al. [9] introduced an exact dynamic stiffness approach for vibration analysis of laminated composite beams with arbitrary ply orientation. Norouzi and Rahmani [10] presented exact analytical solutions for anisotropic conductive heat transfer in composite conical shells.

The components of structures widely used in aircraft, reusable space transportation vehicles, and civil engineering are usually supported by elastic foundations. Therefore, it is necessary to include the effects of elastic foundations for a better understanding of the buckling behaviour and loading carrying capacity of composite structures. Tornabene [11] applied the generalised differential quadrature method to study the dynamic 
behaviour of anisotropic doubly curved shells and panels of revolution with a free-form meridian resting on WinklerPasternak elastic foundations. Sofiyev [12] discussed the buckling analysis of composite orthotropic truncated conical shells under a combined axial compression and external pressure and resting on a Pasternak foundation. Zhang and Liew [13] presented a post-buckling analysis of functionally graded carbon nanotube reinforced composite plates resting on Pasternak foundations. Joodaky and Joodaky [14] introduced a semi-analytical closed-form solution for governing equations of thin skew plates with various combinations of clamp, free, and simple supports subjected to uniform loading rested on the elastic foundations of Winkler and Pasternak. Tornabene et al. $[15,16]$ presented the static and dynamic analyses of laminated doubly curved shells and panels of revolution resting on Winkler-Pasternak elastic foundations using the generalised differential quadrature method. Lei et al. [17] studied the buckling behaviour of functionally graded carbon nanotube reinforced composite thick skew plates resting on Pasternak foundations based on an element-free approach.

A three-phase composite is a material consisting of a matrix, reinforced fibres, and particles. The main role of reinforced fibres is to improve the strength of the material, while that of the particles is to prevent cracking and plasticity. The accurate eccentric impact analysis of preloaded shape memory alloy composite plates using novel mixed-order hyperbolic global-local theory is presented by Shariyat and Hosseini [18]. Almeyda et al. [19] applied the asymptotic homogenisation method and derivation of anti-plane effective properties for three-phase magneto-electro-elastic fibre unidirectional reinforced composites with parallelogram cell symmetry. Chung et al. [20] presented an investigation of polymeric composite films using modified $\mathrm{TiO}_{2}$ nanoparticles for organic light-emitting diodes. Duc and Minh [21] determined the bending deflection of three-phase polymer composite plates consisting of reinforced glass fibres and titanium dioxide $\left(\mathrm{TiO}_{2}\right)$ particles. Hao et al. [22] dealt with the threedimensional simulation of microstructure evolution for three-phase nanocomposite ceramic tool materials. Zhou et al. [23] investigated the elastic modulus of concrete as a three-phase heterogeneous composite. A large deformation, isogeometric rotation-free Kirchhoff-Love shell formulation is equipped with a damage model to efficiently and accurately simulate progressive failure in laminated composite structures in the work of Deng et al. [24]. Recently, Duc and Thu [25] and Duc et al. [26] studied the non-linear static analysis of three-phase polymer composite plates under thermal and mechanical loads. Duc et al.
[27] also presented an investigation on the non-linear dynamic response and vibration of imperfect laminated three-phase polymer nanocomposite cylindrical panels resting on elastic foundations and subjected to hydrodynamic loads (without temperature).

This paper presents an analytical approach to investigate the non-linear dynamic response and vibration of an imperfect three-phase laminated polymer nanocomposite cylindrical panel resting on elastic foundations in thermal environments. Based on the classical laminated shell theory (CLST) and stress function taking into account geometrical non-linearity, initial geometrical imperfection, a Pasternak-type elastic foundation, and temperature, the governing equations of the three-phase composite cylindrical panel are derived. Numerical results for the dynamic response and vibration of the three-phase laminated polymer composite cylindrical panel are obtained by using the Runge-Kutta method. The influences of fibres and nanoparticles, material and geometrical properties, foundation stiffness, imperfection, and temperature on the non-linear dynamic response of the three-phase laminated composite cylindrical panel are discussed in detail.

\section{Determination of the elastic modules of the three-phase composite}

In this paper, the algorithm that was successfully applied in Refs. [25-27] to determine the elastic modules of the three-phase composite has been used. According to this algorithm, the elastic modules of three-phase composites are estimated using two theoretical models of the twophase composite consecutively: $\mathrm{nDm}=\mathrm{Om}+\mathrm{nD}$ [25-27]. This paper considers a three-phase composite reinforced with particles and unidirectional fibres, so the model of the problem will be $1 \mathrm{Dm}=\mathrm{Om}+1 \mathrm{D}$. Firstly, the modules of the effective matrix Om, which are called the "effective modules," are calculated. In this step, the effective matrix consists of the original matrix and added nanoparticles. It is considered to be homogeneous, isotropic, and to have two effective elastic modules. The next step is estimating the elastic modules for a composite material consisting of the effective matrix and unidirectional reinforced fibres.

Assuming that all the component phases (matrix, fibre, and particles) are homogeneous and isotropic, we will use $E_{m}, E_{a}, E_{c} ; v_{m}, v_{a}, v_{c} ; \psi_{m}, \psi_{a}, \psi_{c}$ to denote Young's modulus, Poisson's ratio, and the volume fraction for the matrix, fibre, and nanoparticles, respectively. Following 
Refs. [20, 21], the effective modules for the two-phase composite (polymer matrix reinforced by nanoparticles) can be obtained as shown below:

$$
\begin{gathered}
\bar{G}=G_{m} \frac{1-\psi_{c}\left(7-5 v_{m}\right) H}{1+\psi_{c}\left(8-10 v_{m}\right) H}, \\
\bar{K}=K_{m} \frac{1+4 \psi_{c} G_{m} L\left(3 K_{m}\right)^{-1}}{1-4 \psi_{c} G_{m} L\left(3 K_{m}\right)^{-1}},
\end{gathered}
$$

where

$$
L=\frac{K_{c}-K_{m}}{K_{c}+\frac{4 G_{m}}{3}}, \quad H=\frac{G_{m} / G_{c}-1}{8-10 v_{m}+\left(7-5 v_{m}\right) \frac{G_{m}}{G_{c}}} .
$$

$\bar{E}, \bar{v}$ can be calculated from $(\bar{G}, \bar{K})$ as below:

$$
\bar{E}=\frac{9 \bar{K} \bar{G}}{3 \bar{K}+\bar{G}}, \quad \bar{v}=\frac{3 \bar{K}-2 \bar{G}}{6 \bar{K}-2 \bar{G}} .
$$

The elastic moduli for a three-phase composite reinforced with unidirectional fibres in next step, is chosen to be calculated using Vanin's formulas [28], as

$$
\begin{aligned}
& E_{11}=\psi_{a} E_{a}+\left(1-\psi_{a}\right) \bar{E}+\frac{8 \bar{G} \psi_{a}\left(1-\psi_{a}\right)\left(v_{a}-\bar{v}\right)}{2-\psi_{a}+\bar{X} \psi_{a}+\left(1-\psi_{a}\right)\left(x_{a}-1\right) \frac{\bar{G}}{G_{a}}}, \\
& E_{22}=\left(\frac{v_{21}^{2}}{E_{11}}+\frac{1}{8 \bar{G}}\left[\frac{2\left(1-\psi_{a}\right)(\bar{x}-1)+\left(x_{a}-1\right)\left(\bar{x}-1+2 \psi_{a}\right) \frac{\bar{G}}{G_{a}}}{2-\psi_{a}+\bar{x} \psi_{a}+\left(1-\psi_{a}\right)\left(x_{a}-1\right) \frac{\bar{G}}{G_{a}}}\right.\right. \\
& \left.\left.+2 \frac{\bar{\chi}\left(1-\psi_{a}\right)+\left(1+\psi_{a} \bar{x}\right) \frac{\bar{G}}{G_{a}}}{\bar{x}+\psi_{a}+\left(1-\psi_{a}\right) \frac{\bar{G}}{G_{a}}}\right]\right\}, \\
& G_{12}=\bar{G} \frac{1+\psi_{a}+\left(1-\psi_{a}\right) \frac{\bar{G}}{G_{a}}}{1-\psi_{a}+\left(1+\psi_{a}\right) \frac{\bar{G}}{G_{a}}}, G_{23}=\bar{G} \frac{\bar{x}+\psi_{a}+\left(1-\psi_{a}\right) \frac{\bar{G}}{G_{a}}}{\left(1-\psi_{a}\right) \bar{x}+\left(1+\bar{x} \psi_{a}\right) \frac{\bar{G}}{G_{a}}}, \\
& \frac{v_{23}}{E_{22}}=-\frac{v_{21}^{2}}{E_{11}}+\frac{1}{8 \bar{G}}\left[2 \frac{\left(1-\psi_{a}\right) \bar{X}+\left(1+\psi_{a} \bar{x}\right) \frac{\bar{G}}{G_{a}}}{\bar{x}+\psi_{a}+\left(1-\psi_{a}\right) \frac{\bar{G}}{G_{a}}}\right. \\
& \left.-\frac{2\left(1-\psi_{a}\right)(\bar{x}-1)+\left(x_{a}-1\right)\left(\bar{x}-1+2 \psi_{a}\right) \frac{\bar{G}}{G_{a}}}{2-\psi_{a}+\bar{x} \psi_{a}+\left(1-\psi_{a}\right)\left(x_{a}-1\right) \frac{\bar{G}}{G_{a}}}\right],
\end{aligned}
$$

$$
v_{21}=\bar{v}-\frac{(\bar{x}+1)\left(\bar{v}-v_{a}\right) \psi_{a}}{2-\psi_{a}+\bar{x} \psi_{a}+\left(1-\psi_{a}\right)\left(x_{a}-1\right) \frac{\bar{G}}{G_{a}}},
$$

in which

$$
\bar{x}=3-4 \bar{v}, \quad x_{a}=3-4 v_{a} .
$$

Similar to the elastic modulus, the thermal expansion coefficient of the three-phase composite materials was also identified in two steps. First, to determine the coefficient of thermal expansion of the effective matrix [29]:

$$
\alpha^{*}=\alpha_{m}+\left(\alpha_{c}-\alpha_{m}\right) \frac{K_{c}\left(3 K_{m}+4 G_{m}\right) \psi_{c}}{K_{m}\left(3 K_{c}+4 G_{m}\right)+4\left(K_{c}-K_{m}\right) G_{m} \psi_{c}},
$$

in which $\alpha^{*}$ is the effective thermal expansion coefficient of the effective matrix, and $\alpha_{m}, \alpha_{c}$, are the thermal expansion coefficients of the original matrix and particle, respectively. Then, determining two coefficients of thermal expansion of the three-phase composite using formulas from [28] of Vanin gives

$$
\begin{aligned}
& \alpha_{1}=\alpha^{*}-\left(\alpha^{*}-\alpha_{a}\right) \psi_{a} E_{1}^{-1}\left[E_{a}+\frac{8 G_{a}\left(v_{a}-v\right)\left(1-\psi_{a}\right)\left(1+v_{a}\right)}{2-\psi_{a}+\bar{x} \psi_{a}+\left(1-\psi_{a}\right)\left(x_{a}-1\right) \frac{\bar{G}}{G_{a}}}\right], \\
& \alpha_{2}=\alpha^{*}+\left(\alpha^{*}-\alpha_{1}\right) v_{21}-\left(\alpha-\alpha_{a}\right)\left(1+v_{a}\right) \frac{v-v_{21}}{v-v_{a}} .
\end{aligned}
$$

\section{Governing equations}

Consider a three-phase composite cylindrical panel as shown in Figure 1. The cylindrical panel is referred to a Cartesian coordinate system $x, y, z$, where $x y$ is the midplane of the cylindrical panel and $z$ is the thickness coordinator $(-h / 2 \leq z \leq h / 2)$. The radii of curvatures, length, width, and total thickness of the cylindrical panel are $R$, $a, b$, and $h$, respectively.

The three-phase composite cylindrical panel-foundation interaction is represented by the Pasternak model as

$$
q_{e}=k_{1} w-k_{2} \nabla^{2} w
$$

where $\nabla^{2}=\partial^{2} / \partial x^{2}+\partial^{2} / \partial y^{2}, \quad w$ is the deflection of the cylindrical panel, $k_{1}$ is the Winkler foundation modulus, and $k_{2}$ is the shear layer foundation stiffness of the Pasternak model.

In this study, the CLST is used to establish the governing equations and to determine the non-linear response of the composite cylindrical panels. 


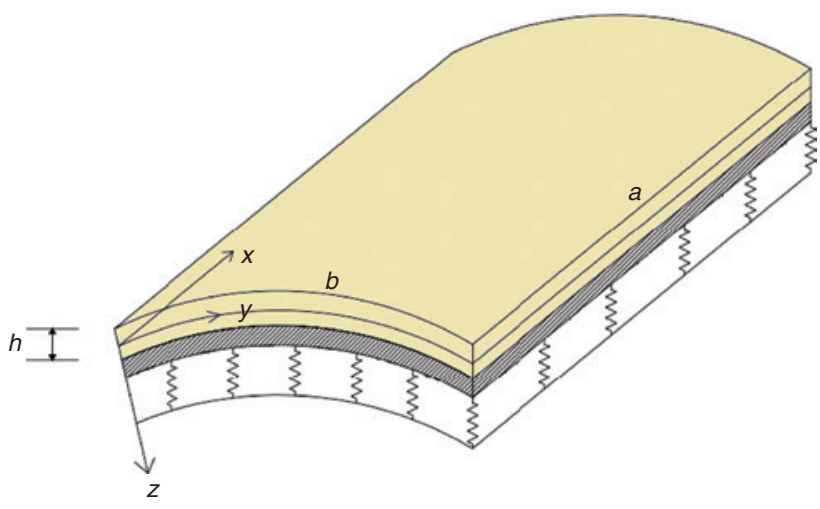

Figure 1: Geometry and coordinate system of the three-phase laminated nanocomposite cylindrical panel on elastic foundations.

Taking into account the von Karman non-linearity, the strain-displacement relations are

$$
\left(\begin{array}{c}
\varepsilon_{x} \\
\varepsilon_{y} \\
\gamma_{x y}
\end{array}\right)=\left(\begin{array}{c}
\varepsilon_{x}^{0} \\
\varepsilon_{y}^{0} \\
\gamma_{x y}^{0}
\end{array}\right)+z\left(\begin{array}{c}
k_{x} \\
k_{y} \\
k_{x y}
\end{array}\right)
$$

where

$$
\left(\begin{array}{c}
\varepsilon_{x}^{0} \\
\varepsilon_{y}^{0} \\
\gamma_{x y}^{0}
\end{array}\right)=\left(\begin{array}{c}
u_{, x}+w_{, x}^{2} / 2 \\
v_{, y}-w / R+w_{, y}^{2} / 2 \\
u_{, y}+v_{, x}+w_{, x} w_{, y}
\end{array}\right),\left(\begin{array}{c}
k_{x} \\
k_{y} \\
k_{x y}
\end{array}\right)=\left(\begin{array}{c}
-w_{, x x} \\
-w_{, y y} \\
-2 w_{, x y}
\end{array}\right),
$$

in which $u, v$ are the displacement components along the $x, y$ directions, respectively.

Hooke's law for a laminated composite cylindrical panel is defined as

$$
\left(\begin{array}{l}
\sigma_{x} \\
\sigma_{y} \\
\sigma_{x y}
\end{array}\right)_{k}=\left(\begin{array}{lll}
Q_{11}^{\prime} & Q_{12}^{\prime} & Q_{16}^{\prime} \\
Q_{12}^{\prime} & Q_{22}^{\prime} & Q_{26}^{\prime} \\
Q_{16}^{\prime} & Q_{26}^{\prime} & Q_{66}^{\prime}
\end{array}\right)_{k}\left(\begin{array}{l}
\varepsilon_{x}-\alpha_{1} \Delta T \\
\varepsilon_{y}-\alpha_{2} \Delta T \\
\gamma_{x y}
\end{array}\right)_{k},
$$

in which $k$ is the number of layers and

$Q_{11}^{\prime}=Q_{11} \cos ^{4} \theta+Q_{22} \sin ^{4} \theta+2\left(Q_{12}+2 Q_{66}\right) \sin ^{2} \theta \cos ^{2} \theta$,

$Q_{12}^{\prime}=Q_{12}\left(\cos ^{4} \theta+\sin ^{4} \theta\right)+\left(Q_{11}+Q_{22}-4 Q_{66}\right) \sin ^{2} \theta \cos ^{2} \theta$,

$Q_{12}^{\prime}=Q_{12}\left(\cos ^{4} \theta+\sin ^{4} \theta\right)+\left(Q_{11}+Q_{22}-4 Q_{66}\right) \sin ^{2} \theta \cos ^{2} \theta$,

$Q_{16}^{\prime}=\left(Q_{12}-Q_{22}+2 Q_{66}\right) \sin ^{3} \theta \cos \theta+\left(Q_{11}-Q_{12}-2 Q_{66}\right) \sin \theta \cos ^{3} \theta$,

$Q_{22}^{\prime}=Q_{11} \sin ^{4} \theta+Q_{22} \cos ^{4} \theta+2\left(Q_{12}+2 Q_{66}\right) \sin ^{2} \theta \cos ^{2} \theta$,

$Q_{26}^{\prime}=\left(Q_{11}-Q_{12}-2 Q_{66}\right) \sin ^{3} \theta \cos \theta+\left(Q_{12}-Q_{22}+2 Q_{66}\right) \sin \theta \cos ^{3} \theta$,

$Q_{66}^{\prime}=Q_{66}\left(\sin ^{4} \theta+\cos ^{4} \theta\right)+\left[Q_{11}+Q_{22}-2\left(Q_{12}+Q_{66}\right)\right] \sin ^{2} \theta \cos ^{2} \theta$,

(13) as well as

$$
\begin{aligned}
& Q_{11}=\frac{E_{11}}{1-\frac{E_{22}}{E_{11}} v_{12}^{2}}=\frac{E_{11}}{1-v_{12} v_{21}}, Q_{22}=\frac{E_{22}}{1-\frac{E_{22}}{E_{11}} v_{12}^{2}}=\frac{E_{22}}{E_{11}} Q_{11}, \\
& Q_{12}=\frac{v_{21} E_{11}}{1-\frac{E_{22}}{E_{11}} v_{12}^{2}}=v_{21} Q_{11}=v_{12} Q_{22}, Q_{66}=G_{12},
\end{aligned}
$$

where $\theta$ is the angle between the fibre direction and the coordinate system. The force and moment resultants of the laminated composite cylindrical panels are determined by

$$
\begin{aligned}
& N_{i}=\sum_{k=1}^{n} \int_{h_{k-1}}^{h_{k}}\left[\sigma_{i}\right]_{k} \mathrm{~d} z, i=x, y, x y, \\
& M_{i}=\sum_{k=1}^{n} \int_{h_{k-1}}^{h_{k}} z\left[\sigma_{i}\right]_{k} \mathrm{~d} z, i=x, y, x y .
\end{aligned}
$$

Substitution of Eq. (10) into Eq. (12) and the result into Eq. (15) gives the constitutive relations as

$$
\begin{aligned}
& \left(N_{x}, N_{y}, N_{x y}\right)=\left(A_{11}, A_{12}, A_{16}\right) \varepsilon_{x}^{0}+\left(A_{12}, A_{22}, A_{26}\right) \varepsilon_{y}^{0} \\
& +\left(A_{16}, A_{26}, A_{66}\right) \gamma_{x y}^{0} \\
& +\left(B_{11}, B_{12}, B_{16}\right) k_{x}+\left(B_{12}, B_{22}, B_{26}\right) k_{y}+\left(B_{16}, B_{26}, B_{66}\right) k_{x y} \\
& -\left[\begin{array}{l}
\left(A_{11}, A_{12}, A_{16}\right) \alpha_{1} \\
+\left(A_{12}, A_{22}, A_{26}\right) \alpha_{2}
\end{array}\right] \Delta T, \\
& \left(M_{x}, M_{y}, M_{x y}\right)=\left(B_{11}, B_{12}, B_{16}\right) \varepsilon_{x}^{0}+\left(B_{12}, B_{22}, B_{26}\right) \varepsilon_{y}^{0} \\
& +\left(B_{16}, B_{26}, B_{66}\right) \gamma_{x y}^{0} \\
& +\left(D_{11}, D_{12}, D_{16}\right) k_{x}+\left(D_{12}, D_{22}, D_{26}\right) k_{y}+\left(D_{16}, D_{26}, D_{66}\right) k_{x y} \\
& -\left[\begin{array}{l}
\left(B_{11}, B_{12}, B_{16}\right) \alpha_{1} \\
+\left(B_{12}, B_{22}, B_{26}\right) \alpha_{2}
\end{array}\right] \Delta T \text {. }
\end{aligned}
$$

where

$$
\begin{aligned}
& A_{i j}=\sum_{k=1}^{n}\left(Q_{i j}^{\prime}\right)_{k}\left(h_{k}-h_{k-1}\right), i, j=1,2,6, \\
& B_{i j}=\frac{1}{2} \sum_{k=1}^{n}\left(Q_{i j}^{\prime}\right)_{k}\left(h_{k}^{2}-h_{k-1}^{2}\right) i, j=1,2,6, \\
& D_{i j}=\frac{1}{3} \sum_{k=1}^{n}\left(Q_{i j}^{\prime}\right)_{k}\left(h_{k}^{3}-h_{k-1}^{3}\right) i, j=1,2,6 .
\end{aligned}
$$

The non-linear motion equation of the composite cylindrical panels based on CLST and Volmir's assumption [30] $\left(u<<w, v<<w, \rho_{1} \frac{\partial^{2} u}{\partial t^{2}} \rightarrow 0, \rho_{1} \frac{\partial^{2} v}{\partial t^{2}} \rightarrow 0\right)$ are given by 


$$
\begin{gathered}
N_{x, x}+N_{x y, y}=0 \\
N_{x y, x}+N_{y, y}=0 \\
M_{x, x x}+2 M_{x y, x y}+M_{y, y y}+N_{x} w_{, x x}+2 N_{x y} w_{, x y}+N_{y} w_{, y y}+q-k_{1} w \\
+k_{2} \nabla^{2} w+\frac{N_{y}}{R}=\rho_{1} \frac{\partial^{2} w}{\partial t^{2}}
\end{gathered}
$$

with $\rho_{1}=\rho h$, where $\rho$ is the mass density of the composite cylindrical panels and $q$ is an external pressure uniformly distributed on the surface of the cylindrical panel.

Calculated from Eq. (14), we have

$$
\begin{aligned}
\varepsilon_{x}^{0} & =A_{11}^{*} N_{x}+A_{12}^{*} N_{y}+A_{16}^{*} N_{x y}-B_{11}^{*} k_{x}-B_{12}^{*} k_{y}-B_{16}^{*} k_{x y} \\
& +\Delta T\left(\alpha_{1} D_{11}^{*}+\alpha_{2} D_{12}^{*}\right), \\
\varepsilon_{y}^{0} & =A_{12}^{*} N_{x}+A_{22}^{*} N_{y}+A_{26}^{*} N_{x y}-B_{21}^{*} k_{x}-B_{22}^{*} k_{y}-B_{26}^{*} k_{x y} \\
& +\Delta T\left(\alpha_{1} D_{21}^{*}+\alpha_{2} D_{22}^{*}\right), \\
\gamma_{x y}^{0} & =A_{16}^{*} N_{x}+A_{26}^{*} N_{y}+A_{66}^{*} N_{x y}-B_{16}^{*} k_{x}-B_{26}^{*} k_{y}-B_{66}^{*} k_{x y} \\
& +\Delta T\left(\alpha_{1} D_{16}^{*}+\alpha_{2} D_{26}^{*}\right),
\end{aligned}
$$

where

$$
\begin{aligned}
& A_{11}^{*}=\frac{A_{22} A_{66}-A_{26}^{2}}{\Delta}, A_{12}^{*}=\frac{A_{16} A_{26}-A_{12} A_{66}}{\Delta}, A_{16}^{*}=\frac{A_{12} A_{26}-A_{22} A_{16}}{\Delta}, \\
& A_{22}^{*}=\frac{A_{11} A_{66}-A_{16}^{2}}{\Delta}, A_{26}^{*}=\frac{A_{12} A_{16}-A_{11} A_{26}}{\Delta}, A_{66}^{*}=\frac{A_{11} A_{22}-A_{12}^{2}}{\Delta}, \\
& A_{22}^{*}=\frac{A_{11} A_{66}-A_{16}^{2}}{\Delta}, A_{26}^{*}=\frac{A_{12} A_{16}-A_{11} A_{26}}{\Delta}, A_{66}^{*}=\frac{A_{11} A_{22}-A_{12}^{2}}{\Delta}, \\
& \Delta=A_{11} A_{22} A_{66}-A_{11} A_{26}^{2}+2 A_{12} A_{16} A_{26}-A_{12}^{2} A_{66}-A_{16}^{2} A_{22}, \\
& B_{11}^{*}=A_{11}^{*} B_{11}+A_{12}^{*} B_{12}+A_{16}^{*} B_{16}, B_{12}^{*}=A_{11}^{*} B_{12}+A_{12}^{*} B_{22}+A_{16}^{*} B_{26}, \\
& B_{16}^{*}=A_{11}^{*} B_{16}+A_{12}^{*} B_{26}+A_{16}^{*} B_{66}, B_{21}^{*}=A_{12}^{*} B_{11}+A_{22}^{*} B_{12}+A_{26}^{*} B_{16}, \\
& B_{22}^{*}=A_{12}^{*} B_{12}+A_{22}^{*} B_{22}+A_{26}^{*} B_{26}, B_{26}^{*}=A_{12}^{*} B_{16}+A_{22}^{*} B_{26}+A_{26}^{*} B_{66}, \\
& B_{61}^{*}=A_{16}^{*} B_{11}+A_{26}^{*} B_{12}+A_{66}^{*} B_{16}, B_{62}^{*}=A_{16}^{*} B_{12}+A_{26}^{*} B_{22}+A_{66}^{*} B_{26}, \\
& B_{66}^{*}=A_{16}^{*} B_{16}+A_{26}^{*} B_{26}+A_{66}^{*} B_{66} .
\end{aligned}
$$

Substituting once again Eq. (19) into the expression of $M_{i j}$ in Eq. (16), then $M_{i j}$ into Eq. (18c) leads to

$$
\begin{aligned}
& N_{x, x}+N_{x y, y}=0, \\
& N_{x y, x}+N_{y, y}=0 \\
& P_{1} f_{, x x x x}+P_{2} f_{, y y y y}+P_{3} f_{, x x y y}+P_{4} f_{, x x x y}+P_{5} f_{, x y y y}+P_{6} w_{, x x x x}+P_{7} w_{, y y y y} \\
& +P_{8} w_{, x x y y}+P_{9} w_{, x x x y}+P_{10} w_{, x y y y}+N_{x} w_{, x x}+2 N_{x y} w_{, x y}+N_{y} w_{, y y} \\
& +q-k_{1} w+k_{2} \nabla^{2} w+\frac{N_{y}}{R}=\rho_{1} \frac{\partial^{2} w}{\partial t^{2}}
\end{aligned}
$$

where

$$
\begin{aligned}
P_{1} & =B_{21}^{*}, P_{2}=B_{12}^{*}, P_{3}=B_{11}^{*}+B_{22}^{*}-2 B_{66}^{*}, P_{4}=2 B_{26}^{*}-B_{61}^{*}, \\
P_{5} & =2 B_{16}^{*}-B_{62}^{*}, P_{6}=B_{11} B_{11}^{*}+B_{12} B_{21}^{*}+B_{16} B_{61}^{*}-D_{11}, \\
P_{7} & =B_{12} B_{12}^{*}+B_{22} B_{22}^{*}+B_{26} B_{62}^{*}-D_{22}, \\
P_{8} & =B_{11} B_{12}^{*}+B_{12} B_{22}^{*}+B_{16} B_{62}^{*}+B_{12} B_{11}^{*}+B_{22} B_{21}^{*}+B_{26} B_{61}^{*}+4 B_{16} B_{16}^{*} \\
& +4 B_{26} B_{26}^{*}+4 B_{66} B_{66}^{*}-4 D_{66}-2 D_{12}, \\
P_{9} & =2\left(B_{11} B_{16}^{*}+B_{12} B_{26}^{*}+B_{16} B_{66}^{*}+B_{16} B_{11}^{*}+B_{26} B_{21}^{*}+B_{66} B_{61}^{*}\right)-4 D_{16}, \\
P_{10} & =2\left(B_{12} B_{16}^{*}+B_{22} B_{26}^{*}+B_{26} B_{66}^{*}+B_{16} B_{12}^{*}+B_{26} B_{22}^{*}+B_{66} B_{62}^{*}\right)-4 D_{26} .
\end{aligned}
$$

$f(x, y)$ is a stress function defined by

$$
N_{x}=f_{, y y}, N_{y}=f_{, x x}, N_{x y}=-f_{, x y} .
$$

For an imperfect laminated composite cylindrical panel, Eq. (21) is modified to

$$
\begin{aligned}
& P_{1} f_{, x x x x}+P_{2} f_{, y y y y}+P_{3} f_{, x x y y}+P_{4} f_{, x x x y}+P_{5} f_{, x y y y}+P_{6} w_{, x x x x}+P_{7} w_{, y y y y} \\
& +P_{8} w_{, x x y y}+P_{9} w_{, x x x y}+P_{10} w_{, x y y y}+f_{, y y}\left(w_{, x x}+w_{, x x}^{*}\right)-2 f_{, x y}\left(w_{, x y}+w_{, x y}^{*}\right) \\
& +f_{, x x}\left(w_{, y y}+w_{, y y}^{*}\right)+q-k_{1} w+k_{2} \nabla^{2} w+\frac{N_{y}}{R}=\rho_{1} \frac{\partial^{2} w}{\partial t^{2}}
\end{aligned}
$$

in which $w^{*}(x, y)$ is a known function representing the initial small imperfection of the cylindrical panel.

The geometrical compatibility equation for an imperfect composite cylindrical panel is written as [25-27]

$$
\begin{aligned}
\varepsilon_{x, y y}^{0}+\varepsilon_{y, x x}^{0}-\gamma_{x y, x y}^{0}= & w_{, x y}^{2}-w_{, x x} w_{, y y}+2 w_{, x y} w_{, x y}^{*}-w_{, x x} w_{, y y}^{*} \\
& -w_{, y y} w_{, x x}^{*}-\frac{w_{, x x}}{R} .
\end{aligned}
$$

From the constitutive relations in Eq. (19), in conjunction with Eq. (23), one can write

$$
\begin{aligned}
\varepsilon_{x}^{0}= & A_{11}^{*} f_{, y y}+A_{12}^{*} f_{, x x}-A_{16}^{*} f_{, x y}-B_{11}^{*} k_{x}-B_{12}^{*} k_{y}-B_{16}^{*} k_{x y} \\
& +\Delta T\left(\alpha_{1} D_{11}^{*}+\alpha_{2} D_{12}^{*}\right), \\
\varepsilon_{y}^{0}= & A_{12}^{*} f_{, y y}+A_{22}^{*} f_{, x x}-A_{26}^{*} f_{, x y}-B_{21}^{*} k_{x}-B_{22}^{*} k_{y}-B_{26}^{*} k_{x y} \\
& +\Delta T\left(\alpha_{1} D_{21}^{*}+\alpha_{2} D_{22}^{*}\right), \\
\gamma_{x y}^{0}= & A_{16}^{*} f_{, y y}+A_{26}^{*} f_{, x x}-A_{66}^{*} f_{, x y}-B_{16}^{*} k_{x}-B_{26}^{*} k_{y}-B_{66}^{*} k_{x y} \\
& +\Delta T\left(\alpha_{1} D_{16}^{*}+\alpha_{2} D_{26}^{*}\right) .
\end{aligned}
$$

Setting Eq. (26) into Eq. (25) gives the compatibility equation of an imperfect composite cylindrical panel as

$$
\begin{aligned}
& A_{22}^{*} f_{, x x x x}+A_{11}^{*} f_{, y y y y}+E_{1} f_{, x x y y}-2 A_{16}^{*} f_{, x y y y}-2 A_{26}^{*} f_{, x y x x}+B_{21}^{*} w_{, x x x x} \\
& +B_{12}^{*} w_{, y y y y}+E_{2} w_{, x x y y}+E_{3} w_{, x y y y}+E_{4} w_{, x y x x}=w_{, x y}^{2}-w_{, x x} w_{, y y}+2 w_{, x y} w_{, x y}^{*} \\
& -w_{, x x} w_{, y y}^{*}-w_{, y y} w_{, x x}^{*}-\frac{w_{, x x}}{R}
\end{aligned}
$$


where

$$
\begin{aligned}
& E_{1}=2 A_{12}^{*}+A_{66}^{*}, E_{2}=B_{11}^{*}+B_{22}^{*}-2 B_{66}^{*}, \\
& E_{3}=2 B_{16}^{*}-B_{26}^{*}, E_{4}=2 B_{26}^{*}-B_{16}^{*} .
\end{aligned}
$$

Equations (24) and (27) are basic equations in terms of variables $w$ and $f$, and they are used to investigate the non-linear dynamic stability of the imperfect three-phase polymer nanocomposite cylindrical panel resting on elastic foundations in thermal environments.

The three-phase composite cylindrical panel considered in this paper is assumed to be simply supported with immovable edges. The boundary conditions are

$$
\begin{aligned}
& w=u=M_{x}=0, N_{x}=N_{x 0} \text { at } x=0, a, \\
& w=u=M_{y}=0, N_{y}=N_{y 0} \text { at } y=0, b .
\end{aligned}
$$

The approximate solutions of $w$ and $w^{*}$ satisfying the boundary conditions in Eq. (29) are assumed to be [25-27]

$$
\left(w, w^{*}\right)=(W, \mu h) \sin \lambda_{m} x \sin \delta_{n} y,
$$

where $\lambda_{m}=\frac{m \pi}{a}, \delta_{n}=\frac{n \pi}{b} m, n$ are the natural numbers of half-waves in the corresponding direction $x, y ; W$ is the amplitude of the deflection; and $\mu$ is the imperfection parameter.

Substituting Eq. (30) into the compatibility equation [Eq. (27)], we define the stress function as

$$
\begin{aligned}
& f=A_{1} \cos 2 \lambda_{m} x+A_{2} \cos 2 \delta_{n} y+A_{3} \sin \lambda_{m} x \sin \delta_{n} y \\
& +A_{4} \cos \lambda_{m} x \cos \delta_{n} y+\frac{1}{2} N_{x 0} y^{2}+\frac{1}{2} N_{y 0} x^{2},
\end{aligned}
$$

with

$$
\begin{aligned}
& A_{1}=\frac{\delta_{n}^{2}}{32 A_{22}^{*} \lambda_{m}^{2}} W(W+2 \mu h), A_{2}=\frac{\lambda_{m}^{2}}{32 A_{11}^{*} \delta_{n}^{2}} W(W+2 \mu h), \\
& A_{3}=\left(\frac{F_{1} F_{2}-F_{3} F_{4}}{F_{2}^{2}-F_{4}^{2}}\right) W, A_{4}=\frac{F_{2} F_{3}-F_{1} F_{4}}{\left(F_{2}^{2}-F_{4}^{2}\right)} W .
\end{aligned}
$$

and $F_{i}(i=1 \div 4)$ is given in the Appendix.

Substitution of Eqs. (30) and (31) into Eq. (24), and applying the Galerkin procedure for the resulting equation yields

$$
\frac{a b}{4}\left[\begin{array}{l}
\left(\frac{F_{1} F_{2}-F_{3} F_{4}}{F_{2}^{2}-F_{4}^{2}}\right)\left(P_{3} \lambda_{m}^{2} \delta_{n}^{2}+P_{1} \lambda_{m}^{4}+P_{2} \delta_{n}^{4}-\frac{\lambda_{m}^{2}}{R}\right) \\
-\frac{F_{2} F_{3}-F_{1} F_{4}}{F_{2}^{2}-F_{4}^{2}}\left(P_{4} \lambda_{m}^{3} \delta_{n}+P_{5} \delta_{n}^{3} \lambda_{m}\right)+P_{6} \lambda_{m}^{4} a b+P_{7} \delta_{n}^{4} a b \\
+P_{8} \lambda_{m}^{2} \delta_{n}^{2}-k_{2}\left(\lambda_{m}^{2}+\delta_{n}^{2}\right)-k_{1}
\end{array}\right] W
$$

$$
\begin{aligned}
& +\left[\frac{\delta_{n}}{6 R A_{22}^{*} \lambda_{m}}-\frac{2}{3}\left(\frac{P_{1}}{A_{22}^{*}}-\frac{P_{2}}{A_{11}^{*}}\right) \delta_{n} \lambda_{m}\right] W(W+2 \mu h) \\
& +\frac{8}{3}\left(\frac{F_{1} F_{2}-F_{3} F_{4}}{F_{2}^{2}-F_{4}^{2}}\right) \lambda_{m} \delta_{n} W(W+\mu h) \\
& -\frac{a b}{64}\left(\frac{\delta_{n}^{4}}{A_{22}^{*}}+\frac{\lambda_{m}^{4}}{A_{11}^{*}}\right) W(W+\mu h)(W+2 \mu h) \\
& -\frac{a b}{4}\left(N_{x 0} \lambda_{m}^{2}+N_{y 0} \delta_{n}^{2}\right)(W+\mu h)+\frac{4 q}{\lambda_{m} \delta_{n}} \\
& +\frac{4 N_{y 0}}{R \lambda_{m} \delta_{n}}=\rho_{1} \frac{\partial^{2} W}{\partial t^{2}} \frac{a b}{4} .
\end{aligned}
$$

The non-linear dynamic responses of the threephase polymer composite cylindrical panels in thermal environments can be obtained by solving this equation, combined with the initial conditions to be assumed as $W(0)=0, \frac{\mathrm{d} W}{\mathrm{~d} t}(0)=0$ by using the fourth-order RungeKutta method.

A three-phase laminated nanocomposite cylindrical panel on elastic foundations with all immovable edges is considered. The cylindrical panel is subjected to uniform external pressure $q$ and simultaneously exposed to thermal environments. The in-plane condition on immovability at all edges, i.e. $u=0$ at $x=0, a$ and $v=0$ at $y=0, b$, is fulfilled in an average sense as

$$
\int_{0}^{b} \int_{0}^{a} \frac{\partial u}{\partial x} \mathrm{~d} x \mathrm{~d} y=0, \int_{0}^{a} \int_{0}^{b} \frac{\partial v}{\partial y} \mathrm{~d} x \mathrm{~d} y=0
$$

From Eqs. (11) and (19), we obtain the following expressions, in which Eq. (23) and imperfection have been included as

$$
\begin{aligned}
& \frac{\partial u}{\partial x}=A_{11}^{*} f_{, y y}+A_{12}^{*} f_{, x x}-A_{16}^{*} f_{, x y}+B_{11}^{*} w_{, x x}+B_{12}^{*} w_{, y y}+2 B_{16}^{*} w_{, x y} \\
& -\frac{w_{, x}^{2}}{2}-w_{, x} w_{, x}^{*}+\Delta T\left(\alpha_{1} D_{11}^{*}+\alpha_{2} D_{12}^{*}\right),
\end{aligned}
$$

$$
\begin{aligned}
& \frac{\partial v}{\partial y}=A_{12}^{*} f_{, y y}+A_{22}^{*} f_{, x x}-A_{26}^{*} f_{, x y}+B_{21}^{*} w_{, x x}+B_{22}^{*} w_{, y y}+2 B_{26}^{*} w_{, x y} \\
& +\Delta T\left(\alpha_{1} D_{21}^{*}+\alpha_{2} D_{22}^{*}\right)+\frac{w}{R}-\frac{w_{, y}^{2}}{2}-w_{, y} w_{, y}^{*} .
\end{aligned}
$$

Substitution of Eqs. (30) and (31) into Eqs. (35) and (36), and then the results into Eq. (34), gives the fictitious edge compressive loads as

$$
\begin{aligned}
& N_{x 0}=J_{1} W+J_{2} W(W+2 \mu h)+J_{3} \Delta T, \\
& N_{y 0}=J_{4} W+J_{5} W(W+2 \mu h)+J_{6} \Delta T .
\end{aligned}
$$


Subsequently, substitution of Eqs. (37) and (38) into Eq. (33) yields

$$
\begin{aligned}
& b_{1} W+b_{2} W(W+\mu h)+b_{3} W(W+2 \mu h)-b_{4} W(W+\mu h)(W+2 \mu h) \\
& +\left[b_{5}(W+\mu h)+b_{6}\right] \Delta T+\frac{4 q}{\lambda_{m} \delta_{n}}=\rho_{1} \frac{\partial^{2} W}{\partial t^{2}} \frac{a b}{4} .
\end{aligned}
$$

where $b_{s}(s=1,2, \ldots, 6)$ are defined in the Appendix.

From Eq. (39), the fundamental frequencies of a perfect cylindrical panel can be determined approximately by an explicit expression as

$$
\omega_{m n}=\sqrt{-\frac{e}{\rho_{1}}}
$$

with $e$ is given in the Appendix.

\section{Results and discussion}

We chose the three-phase polymer composite made of polyester AKAVINA (made in Vietnam), glass fibres (made in Korea), and nano titanium dioxide (made in Australia), with the properties shown in Table 1 [25-27].

The results presented in this section from Eq. (33) correspond to the deformation mode with half-wave numbers $m=n=1$. To determine the influences of fibres and particles, material and geometrical properties, foundation stiffness, imperfection, and temperature on the non-linear dynamic response of the polymer composite cylindrical panel, we consider a five-layer symmetric laminated cylindrical panel with a stacking sequence of [45/-45/0/-45/45]. The mass density of the cylindrical panel is $\rho=1550 \mathrm{~kg} / \mathrm{m}^{3}$.

Table 2 shows the effects of particle volume fraction, fibre volume fraction, and elastic foundations on the natural frequencies of the three-phase laminated polymer composite cylindrical panel. It can be seen that the value of the natural oscillation frequency increases when the values $k_{1}(\mathrm{GPa} / \mathrm{m})$ and $k_{2}(\mathrm{GPa} \cdot \mathrm{m})$ increase. Furthermore,

Table 1: Properties of the component phases for the three-phase polymer composite.

\begin{tabular}{lrr}
\hline Component phase & $\begin{array}{r}\text { Young's modulus } \\
\boldsymbol{E}(\mathrm{GPa})\end{array}$ & $\begin{array}{r}\text { Poisson's } \\
\text { ratio v }\end{array}$ \\
\hline $\begin{array}{l}\text { Matrix polyester AKAVINA } \\
\text { (Vietnam) }\end{array}$ & 1.43 & 0.345 \\
Glass fibre (Korea) & 22 & 0.24 \\
$\begin{array}{l}\text { Nano titanium oxide } \mathrm{TiO}_{2} \\
\text { (Australia) }\end{array}$ & 5.58 & 0.20 \\
\hline
\end{tabular}

Table 2: Effects of particle volume fraction, fibre volume fraction, and elastic foundations on the natural frequencies of the threephase polymer composite cylindrical panel.

\begin{tabular}{lrll}
\hline$\psi_{a}$ & $\boldsymbol{\psi}_{\boldsymbol{c}}$ & \multicolumn{1}{c}{$\boldsymbol{\omega}_{\boldsymbol{L}}(\mathrm{rad} / \mathrm{s})$} & \\
\cline { 3 - 4 } & & $\left(\boldsymbol{k}_{\mathbf{1}}, \boldsymbol{k}_{\mathbf{2}}\right)=(\mathbf{0 . 0 1}, \mathbf{0 . 0 0 3})$ & $\left(\boldsymbol{k}_{\mathbf{1}}, \boldsymbol{k}_{\mathbf{2}}\right)=(\mathbf{0 . 0 2}, \mathbf{0 . 0 0 4})$ \\
\hline 0.2 & 0 & $5.2462 \mathrm{e} 3$ & $6.2451 \mathrm{e} 3$ \\
0.2 & 0.15 & $5.2461 \mathrm{e} 3$ & $6.2450 \mathrm{e} 3$ \\
0.2 & 0.3 & $5.2369 \mathrm{e} 3$ & $6.2373 \mathrm{e} 3$ \\
0.2 & 0.4 & $5.2263 \mathrm{e} 3$ & $6.2284 \mathrm{e} 3$ \\
0 & 0.2 & $5.4984 \mathrm{e} 3$ & $6.4584 \mathrm{e} 3$ \\
0.15 & 0.2 & $5.3090 \mathrm{e} 3$ & $6.2980 \mathrm{e} 3$ \\
0.3 & 0.2 & $5.1171 \mathrm{e} 3$ & $6.1370 \mathrm{e} 3$ \\
0.4 & 0.2 & $4.9977 \mathrm{e} 3$ & $6.0378 \mathrm{e} 3$ \\
\hline
\end{tabular}

the Pasternak elastic foundation influence on the natural oscillation frequency is larger than the Winkler foundation. The natural frequencies of the cylindrical panels are observed to be dependent on the particle volume fraction and fibre volume fraction; they decrease when increasing the particle volume fraction $\psi_{c}$ and fibre volume fraction $\psi_{a}$, and the effect of the fibre on natural frequency is stronger than the particle.

Figure 2 (with glass fibre volume fractions $\psi_{a}=0.2=$ const and nano titanium oxide particle volume fraction $\psi_{c}=0 ; 0.1 ; 0.2$ ) and Figure 3 (with nanoparticle volume fractions $\psi_{c}=0.2=$ const and glass fibre volume fraction $\left.\psi_{a}=0 ; 0.1 ; 0.2\right)$ represent the effects of the particle and the fibre volume fractions on the dynamic response of the three-phase polymer laminated composite cylindrical panel. Obviously, an increase of the particle and fibre densities will decrease the amplitude of the cylindrical panel. However, the effects of the fibres are stronger.

Figures 4-6 illustrate the effects of geometrical dimensions $b / a, b / h, R / h$ on the non-linear dynamic response of the three-phase laminated polymer composite cylindrical panel. As can be seen, the amplitude of the cylindrical panel increases when decreasing the ratios $b / a, b / h$ and increasing the ratio $R / h$.

Figures 7 and 8 show the effect of elastic foundation stiffness on the non-linear dynamic response of the three-phase laminated polymer composite cylindrical panel. We can see that the cylindrical panel fluctuation amplitude decreases when the stiffness $k_{1}$ and $k_{2}$ increase; namely, the amplitude of the cylindrical panel decreases when it rests on elastic foundations, and the beneficial effect of the Pasternak foundation is better than the Winkler one.

Figure 9 gives the effect of initial imperfection on the dynamic response of the three-phase laminated polymer 


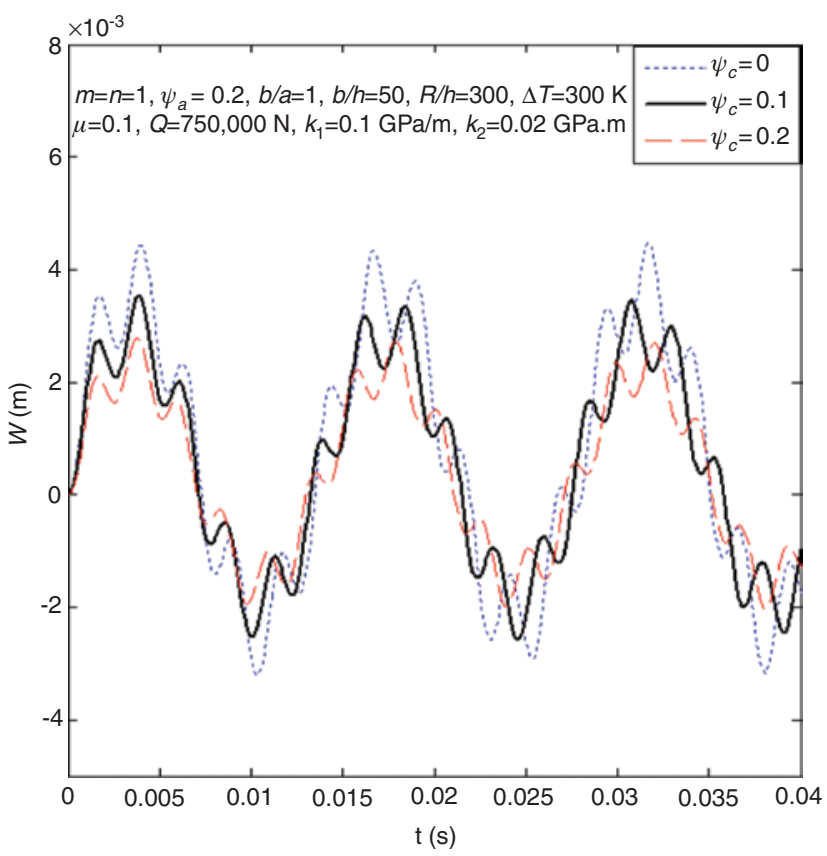

Figure 2: Effects of the particle volume fraction $\psi_{c}$ on the dynamic response of the three-phase laminated polymer composite cylindrical panel.

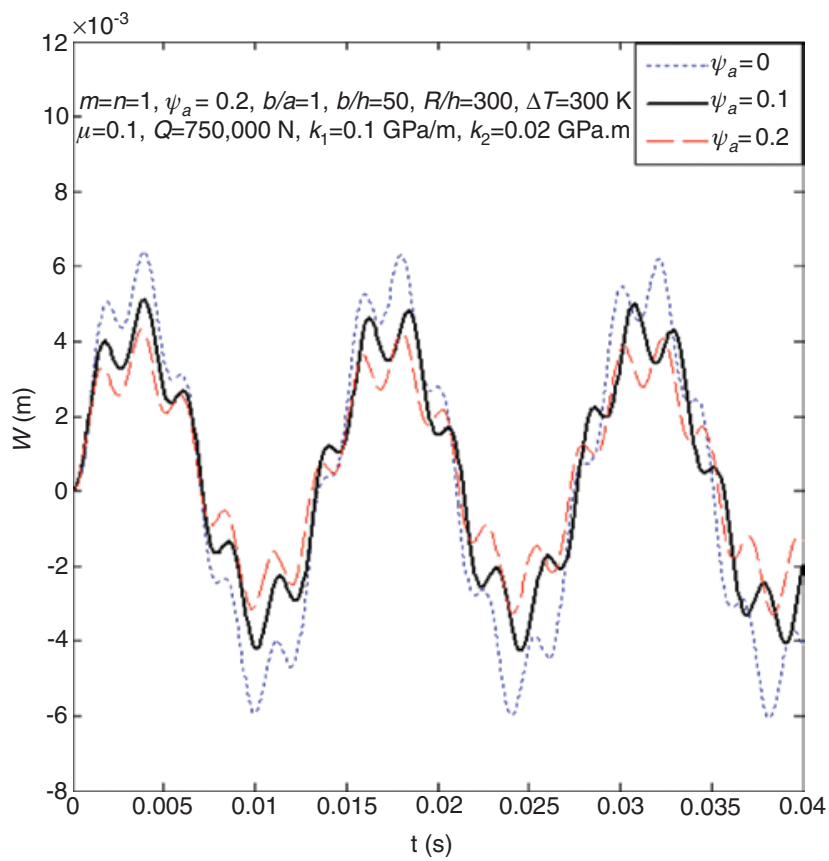

Figure 3: Effects of the fibre volume fraction $\psi_{a}$ on the dynamic response of the three-phase laminated polymer composite cylindrical panel.

composite cylindrical panel. Obviously, the imperfect coefficient has a significant effect on the dynamic response of the cylindrical panel.

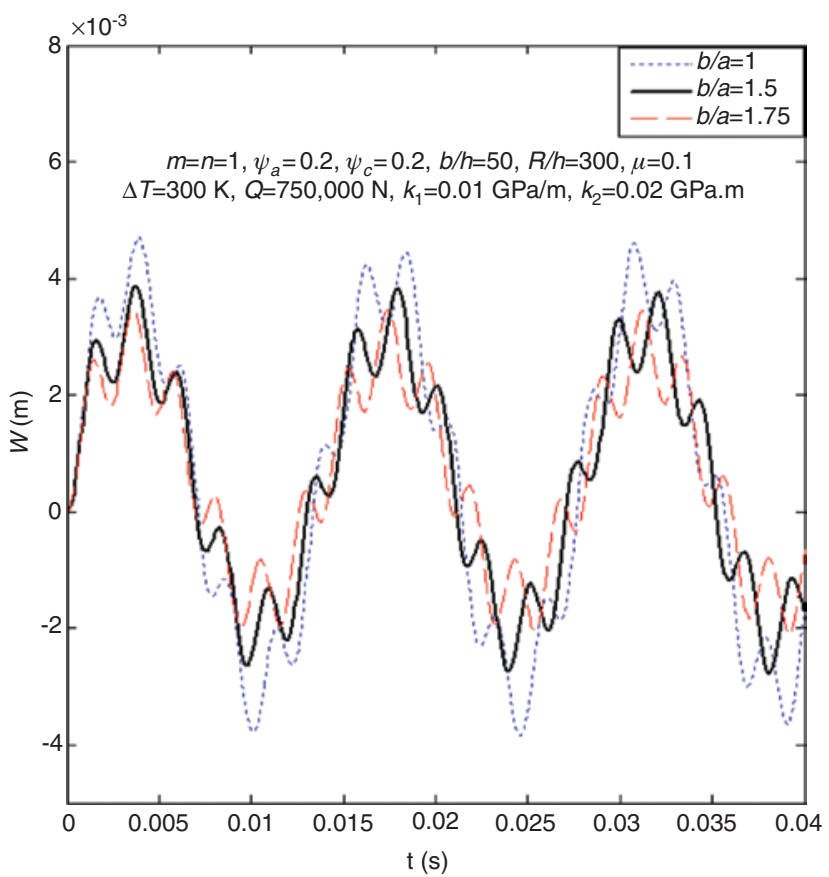

Figure 4: Effect of the $b / a$ ratio on the non-linear dynamic response of the polymer composite cylindrical panel.

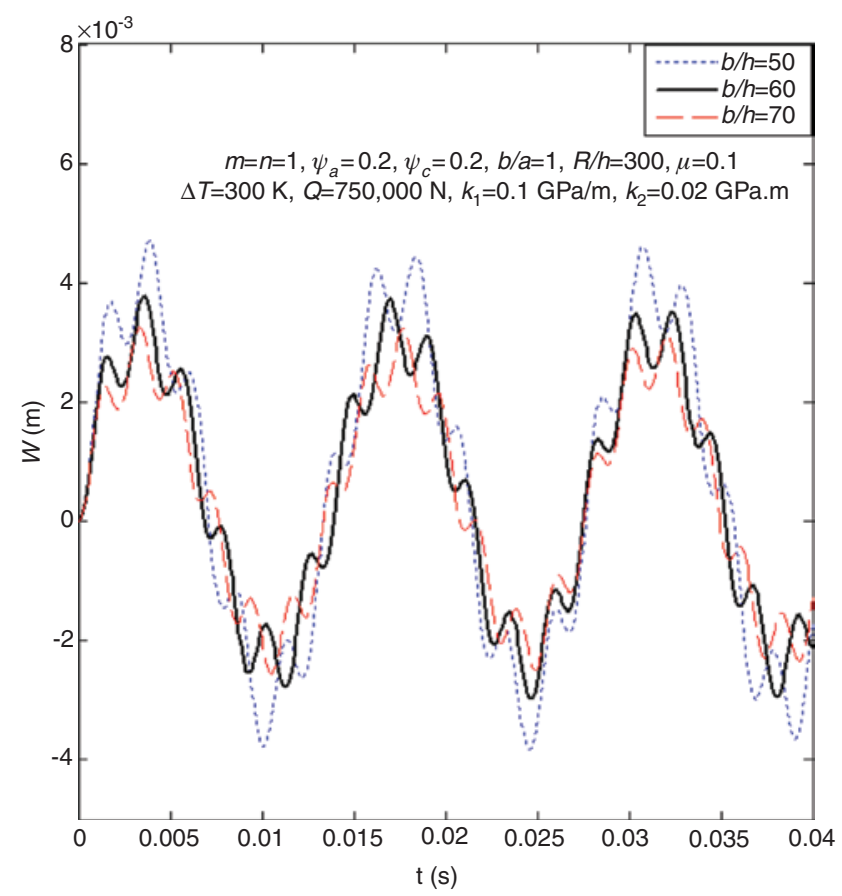

Figure 5: Effect of the $b / h$ ratio on the non-linear dynamic response of the polymer composite cylindrical panel.

Figure 10 shows the effect of temperature increment on the non-linear dynamic response of the three-phase laminated polymer composite cylindrical panel. It can 


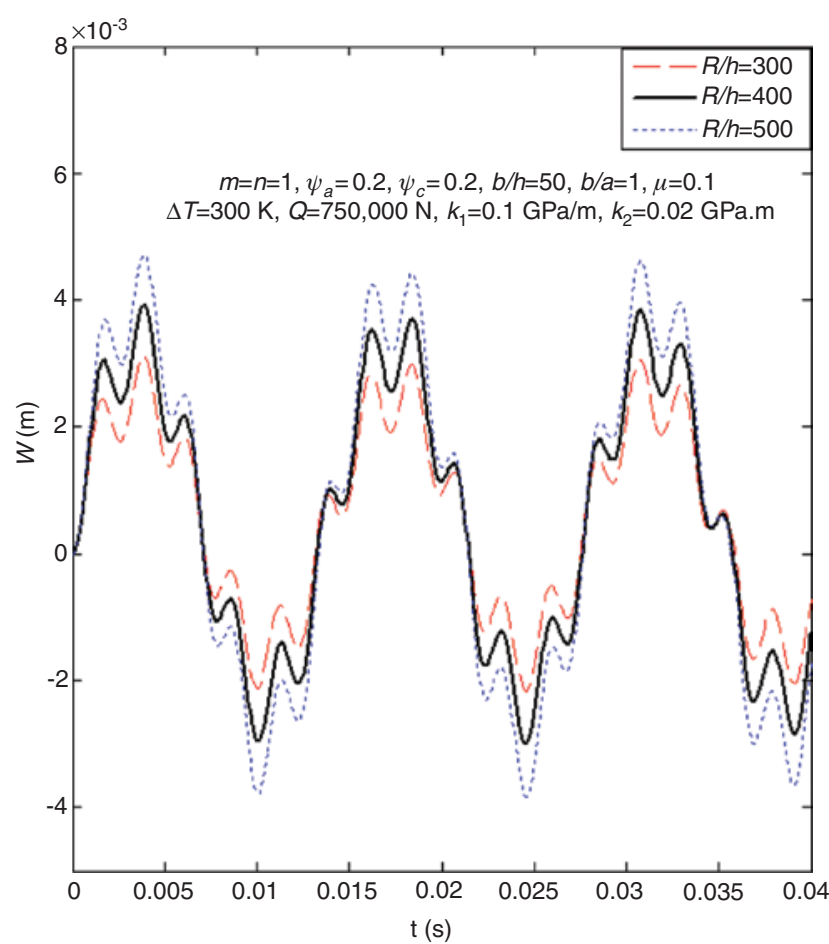

Figure 6: Effect of the $R / h$ ratio on the non-linear dynamic response of the polymer composite cylindrical panel.

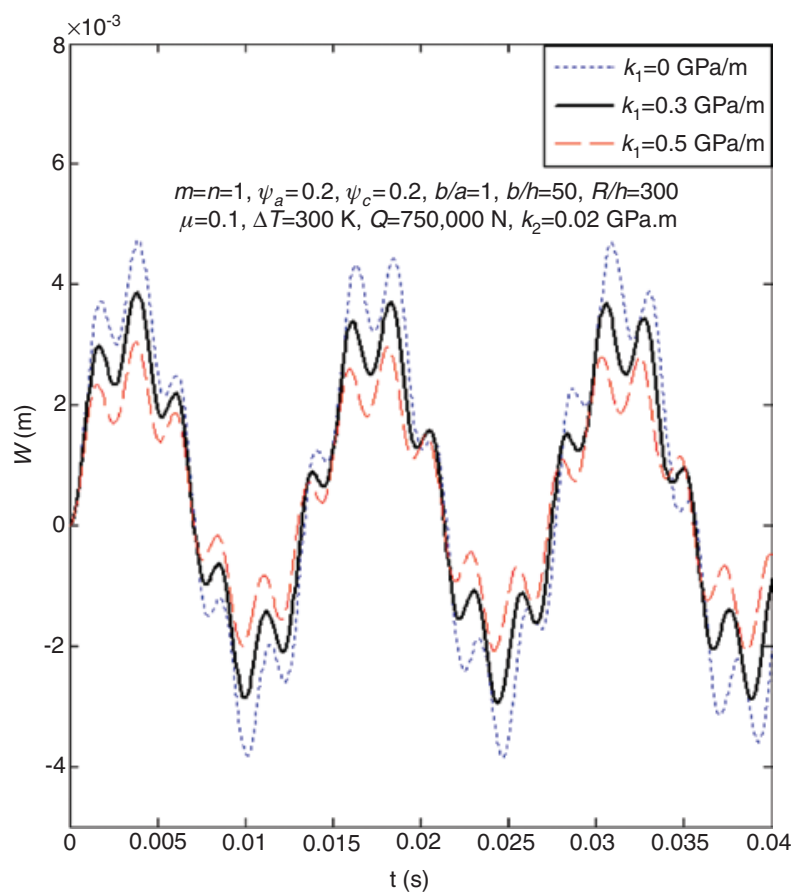

Figure 7: Effect of the linear Winkler foundation on the non-linear dynamic response of the polymer composite cylindrical panel.

be seen that the amplitude of the cylindrical panel will increase and lose stability if the temperature increment increases.

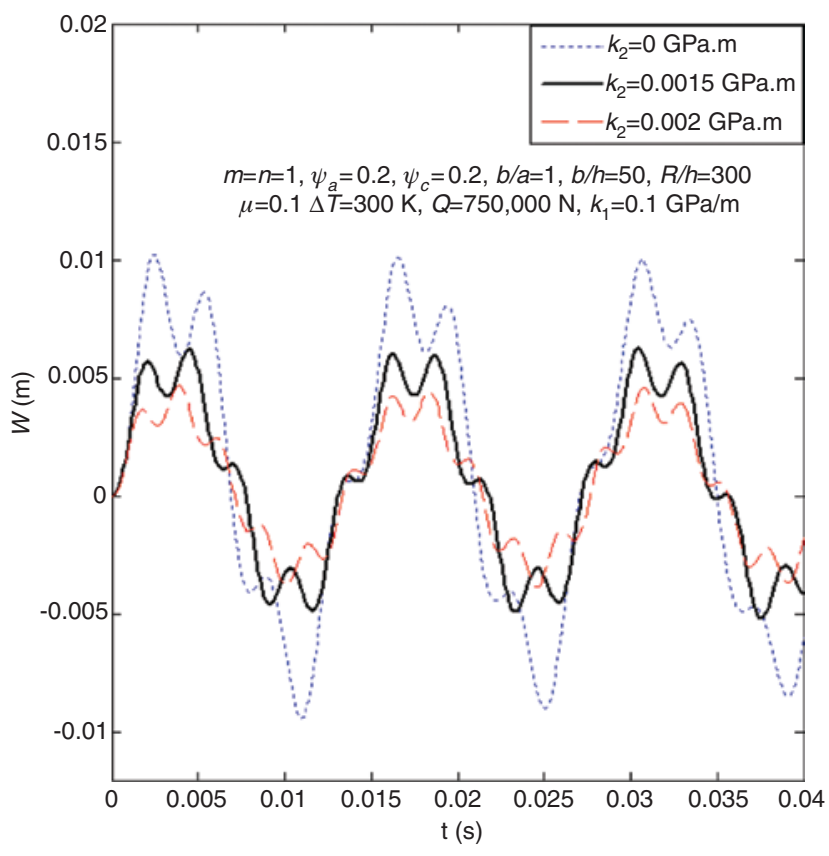

Figure 8: Effect of the Pasternak foundation on the non-linear dynamic response of the polymer composite cylindrical panel.

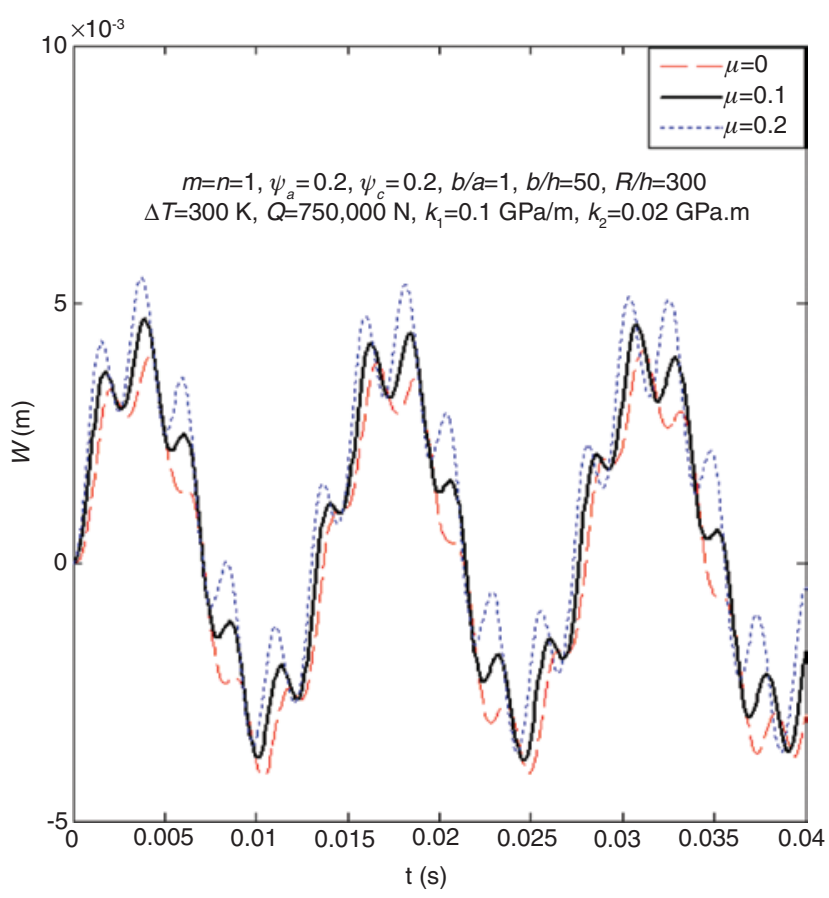

Figure 9: Effect of the imperfection parameter on the non-linear dynamic response of the polymer composite cylindrical panel.

Figure 11 compares the non-linear dynamic response of the three-phase laminated polymer composite cylindrical panel in two cases: the five-layer asymmetric laminated cylindrical panel with a stacking sequence of 


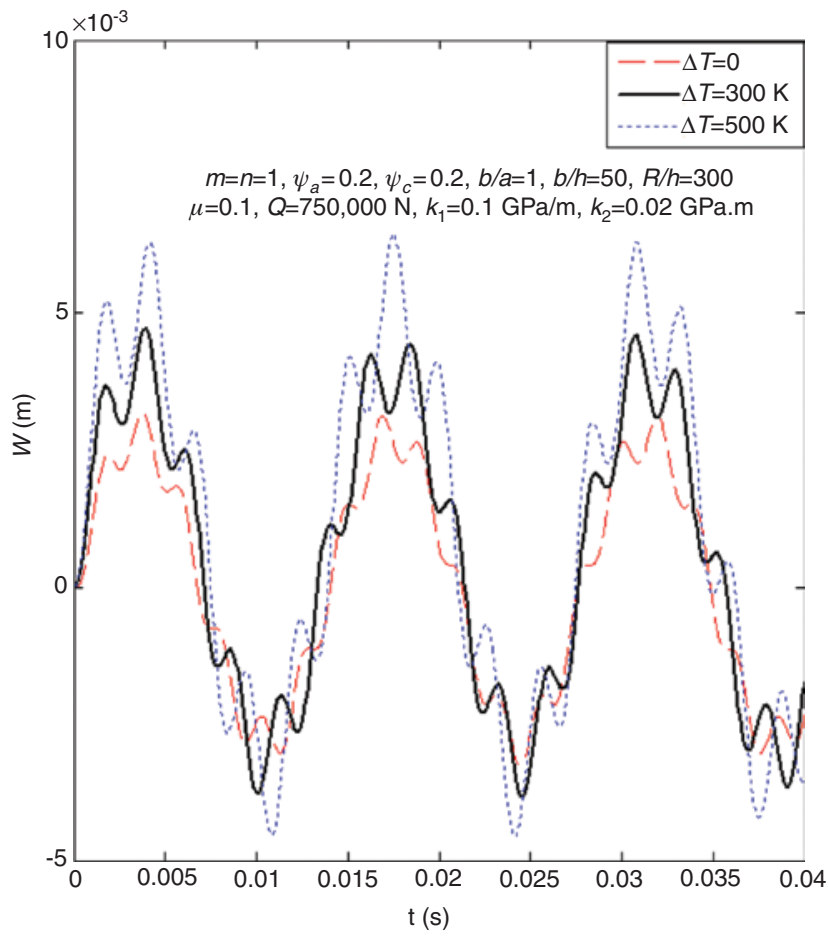

Figure 10: Effects of the temperature increment on the non-linear dynamic response of the polymer composite cylindrical panel.

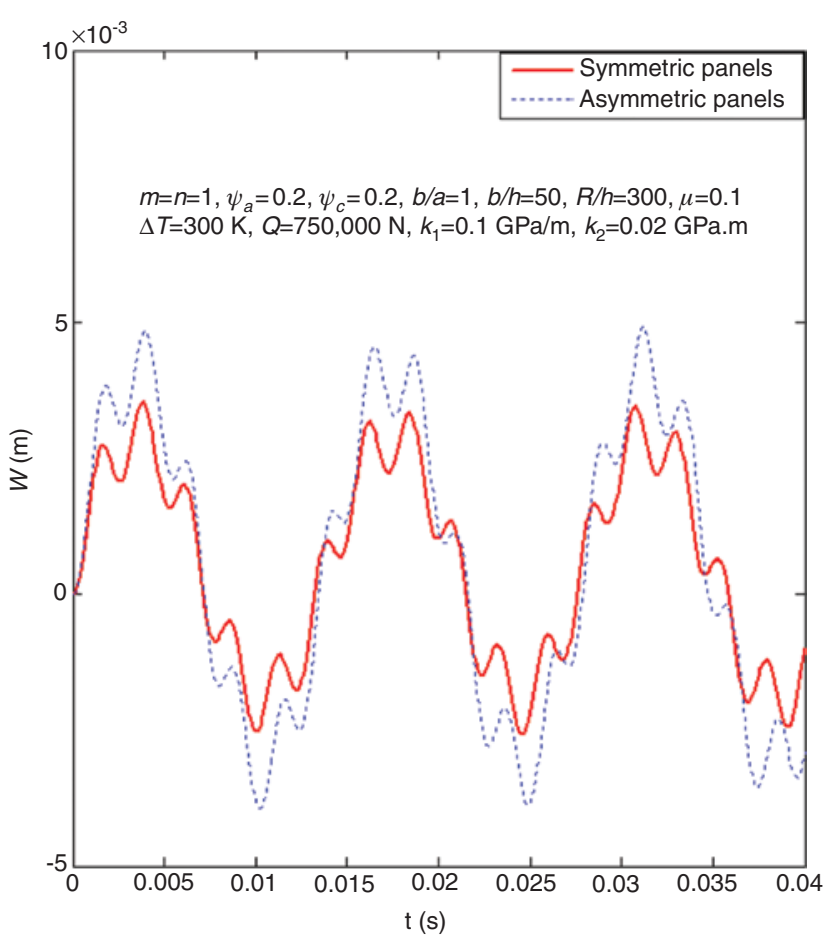

Figure 11: Non-linear dynamic response of the polymer composite cylindrical panel with different fibre angles.
[0/45/45/-45/-45] and the five-layer symmetric laminated cylindrical panel with a stacking sequence of [45/-45/ $0 /-45 / 45]$. This comparison is performed on cylindrical panels with the same ply orientations and the same thickness. The result shows that the amplitude of the asymmetric cylindrical panel is higher than that of the symmetric cylindrical panel.

\section{Conclusions}

This paper presented an analytical approach to investigate the non-linear dynamic response and vibration of an imperfect three-phase laminated nanocomposite cylindrical panel resting on elastic foundations and subjected to uniform external pressure and temperature. The formulations are based on the CLST and stress function taking into account geometrical non-linearity, initial geometrical imperfection, a Pasternak-type elastic foundation, and thermal effects. Numerical results for the dynamic response and vibration of the three-phase polymer composite cylindrical panel with polyester matrix reinforced by glass fibres and nano titanium oxide particles are obtained by using the Runge-Kutta method. The influence of fibre and nanoparticle volume fractions, different fibre angles, material and geometrical properties, imperfection, elastic foundations, and temperature on the non-linear dynamic response of the three-phase laminated polymer composite cylindrical panel are discussed in detail.

Acknowledgments: This work was supported by Grant 107.02-2015.03 in Mechanics of the National Foundation for Science and Technology Development of Vietnam - NAFOSTED and Grant of Newton Fund (UK) Code NRCP1516/1/68. The authors are grateful for this support.

\section{Appendix}

$$
\begin{aligned}
& F_{1}=\left(\frac{\lambda_{m}^{2}}{R}-B_{21}^{*} \lambda_{m}^{4}-B_{12}^{*} \delta_{n}^{4}-E_{2} \lambda_{m}^{2} \delta_{n}^{2}\right), F_{2}=\left(A_{22}^{*} \lambda_{m}^{4}+A_{11}^{*} \delta_{n}^{4}+E_{1} \lambda_{m}^{2} \delta_{n}^{2}\right), \\
& F_{3}=\left(E_{3} \delta_{n}^{3} \lambda_{m}+E_{4} \lambda_{m}^{3} \delta_{n}\right), F_{4}=\left(2 A_{16}^{*} \delta_{n}^{3} \lambda_{m}+2 A_{26}^{*} \lambda_{m}^{3} \delta_{n}\right),
\end{aligned}
$$




$$
b_{1}=\left\{\begin{array}{c}
{\left[\begin{array}{c}
\left(\frac{F_{1} F_{2}-F_{3} F_{4}}{F_{2}^{2}-F_{4}^{2}}\right)\left(P_{3} \lambda_{m}^{2} \delta_{n}^{2}+P_{1} \lambda_{m}^{4}+P_{2} \delta_{n}^{4}-\frac{\lambda_{m}^{2}}{R}\right) \\
+P_{6} \lambda_{m}^{4}+P_{7} \delta_{n}^{4}+P_{8} \lambda_{m}^{2} \delta_{n}^{2}-\frac{F_{2} F_{3}-F_{1} F_{4}}{F_{2}^{2}-F_{4}^{2}}\left(P_{4} \lambda_{m}^{3} \delta_{n}+P_{5} \delta_{n}^{3} \lambda_{m}\right) \\
-k_{2}\left(\lambda_{m}^{2}+\delta_{n}^{2}\right)-k_{1}
\end{array}\right]} \\
+\frac{16}{\frac{\lambda_{m}}{\delta_{n}} F_{1} F_{2}-F_{3} F_{4}}-\frac{A_{11}^{*}-F_{4}^{2}}{\left(A_{11}^{*} A_{22}^{*}-A_{12}^{* 2}\right) R \lambda_{m} \delta_{n}} \\
-\frac{\left(A_{16}^{*} A_{12}^{*}-A_{26}^{*} A_{11}^{*}\right)}{\left(A_{11}^{*} A_{22}^{*}-A_{12}^{* 2}\right)} \\
F_{2} F_{3}-F_{1} F_{4} \\
F_{2}^{2}-F_{4}^{2}
\end{array}\right],-
$$$$
b_{2}=\left\{\begin{array}{l}
\frac{2}{3}\left(\frac{F_{1} F_{2}-F_{3} F_{4}}{F_{2}^{2}-F_{4}^{2}}\right) \lambda_{m} \delta_{n}+\frac{\left(A_{12}^{*} B_{22}^{*}-A_{22}^{*} B_{12}^{*}-A_{12}^{*} B_{11}^{*}+A_{11}^{*} B_{21}^{*}\right)}{\left(A_{11}^{*} A_{22}^{*}-A_{12}^{* 2}\right)} \delta_{n} \lambda_{m} \\
+\frac{\left(A_{12}^{*} B_{21}^{*}-A_{22}^{*} B_{11}^{*}\right) \lambda_{m}^{4}+\left(A_{12}^{*} B_{12}^{*}-A_{11}^{*} B_{22}^{*}\right) \delta_{n}^{4}}{\left(A_{11}^{*} A_{22}^{*}-A_{12}^{*}\right) \lambda_{m} \delta_{n}}-\frac{A_{12}^{*} \lambda_{m}^{2}-A_{11}^{*} \delta_{n}^{2}}{\left(A_{11}^{*} A_{22}^{*}-A_{12}^{*}\right) R \lambda_{m} \delta_{n}} \\
+\frac{\left(A_{26}^{*} A_{12}^{*}-A_{22}^{*} A_{16}^{*} \lambda_{m}^{2}+\left(A_{16}^{*} A_{12}^{*}-A_{26}^{*} A_{11}^{*}\right) \delta_{n}^{2} F_{2} F_{3}-F_{1} F_{4}\right.}{F_{2}^{2}-F_{4}^{2}}
\end{array}\right\},
$$$$
b_{3}=\left[\frac{\delta_{n}}{6 R A_{22}^{*} \lambda_{m}}-\frac{2}{3}\left(\frac{P_{1}}{A_{22}^{*}}+\frac{P_{2}}{A_{11}^{*}}\right) \lambda_{m} \delta_{n}-\frac{1}{2 R \lambda_{m} \delta_{n}} \frac{A_{12}^{*} \lambda_{m}^{2}-A_{11}^{*} \delta_{n}^{2}}{\left(A_{11}^{*} A_{22}^{*}-A_{12}^{* 2}\right)}\right],
$$$$
b_{4}=\frac{a b}{64}\left(\frac{\delta_{n}^{4}}{A_{22}^{*}}+\frac{\lambda_{m}^{4}}{A_{11}^{*}}+\frac{2\left(A_{22}^{*} \lambda_{m}^{4}-2 A_{12}^{*} \lambda_{m}^{2} \delta_{n}^{2}+A_{11}^{*} \delta_{n}^{4}\right)}{\left(A_{11}^{*} A_{22}^{*}-A_{12}^{* 2}\right)}\right),
$$$$
b_{5}=\frac{a b}{4} \frac{\left[\left(A_{22}^{*} \lambda_{m}^{2}-A_{12}^{*} \delta_{n}^{2}\right)\left(D_{11}^{*} \alpha_{1}+D_{12}^{*} \alpha_{2}\right)-\left(A_{12}^{*} \lambda_{m}^{2}-A_{11}^{*} \delta_{n}^{2}\right)\left(D_{21}^{*} \alpha_{1}+D_{22}^{*} \alpha_{2}\right)\right]}{\left(A_{11}^{*} A_{22}^{*}-A_{12}^{*}\right)},
$$

$$
b_{6}=\frac{4}{R \lambda_{m} \delta_{n}} \frac{\left[\left(A_{12}^{*} D_{11}^{*}-A_{11}^{*} D_{21}^{*}\right) \alpha_{1}+\left(A_{12}^{*} D_{12}^{*}-A_{11}^{*} D_{22}^{*}\right) \alpha_{2}\right]}{\left(A_{11}^{*} A_{22}^{*}-A_{12}^{* 2}\right)},
$$

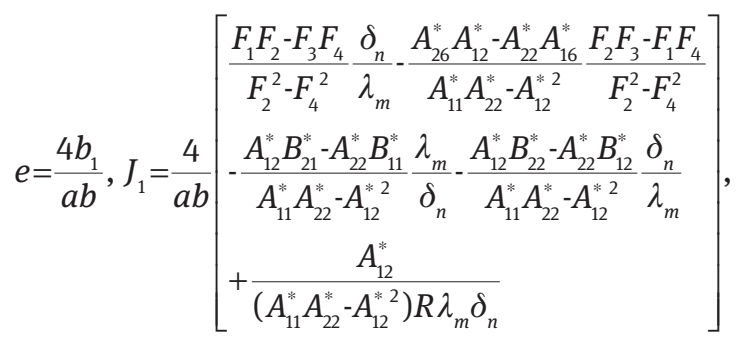

$J_{2}=\frac{1}{8} \frac{A_{22}^{*} \lambda_{m}^{2}-A_{12}^{*} \delta_{n}^{2}}{A_{11}^{*} A_{22}^{*}-A_{12}^{* 2}}, J_{3}=-\frac{\left(A_{22}^{*} D_{11}^{*}-A_{12}^{*} D_{21}^{*}\right) \alpha_{1}+\left(A_{22}^{*} D_{12}^{*}-A_{12}^{*} D_{22}^{*}\right) \alpha_{2}}{A_{11}^{*} A_{22}^{*}-A_{12}^{* 2}}$,

$$
\begin{gathered}
J_{4}=\frac{4}{a b}\left[\begin{array}{l}
\frac{\lambda_{m}}{\delta_{n}} \frac{F_{1} F_{2}-F_{3} F_{4}}{F_{2}^{2}-F_{4}^{2}}-\frac{A_{16}^{*} A_{12}^{*}-A_{26}^{*} A_{11}^{*} F_{2} F_{3}-F_{1} F_{4}}{A_{11}^{*} A_{22}^{*}-A_{12}^{* 2}} \frac{A_{12}^{*} B_{11}^{*}-A_{11}^{*} B_{21}^{*}-F_{4}^{2}}{A_{11}^{*} A_{22}^{*}-A_{12}^{* 2}} \frac{\lambda_{m}}{\delta_{n}} \\
-\frac{A_{12}^{*} B_{12}^{*}-A_{11}^{*} B_{22}^{*}}{A_{11}^{*} A_{22}^{*}-A_{12}^{* 2}} \frac{\delta_{n}}{\lambda_{m}}-\frac{A_{11}^{*}}{\left(A_{11}^{*} A_{22}^{*}-A_{12}^{* 2}\right) R \lambda_{m} \delta_{n}}
\end{array}\right], \\
J_{5}=-\frac{1}{8} \frac{A_{12}^{*} \lambda_{m}^{2}-A_{11}^{*} \delta_{n}^{2}}{A_{11}^{*} A_{22}^{*}-A_{12}^{* 2}}, J_{6}=\frac{\left(A_{12}^{*} D_{11}^{*}-A_{11}^{*} D_{21}^{*}\right) \alpha_{1}+\left(A_{12}^{*} D_{12}^{*}-A_{11}^{*} D_{22}^{*}\right) \alpha_{2}}{A_{11}^{*} A_{22}^{*}-A_{12}^{* 2}} .
\end{gathered}
$$

\section{References}

[1] Jin T, Ha NS, Le VT, Goo NS, Jeon HC. Compos. Struct. 2015, 123, 420-429.

[2] Lei ZX, Zhang LW, Liew KM. Compos. Struct. 2015, 127, 245-259.

[3] Rossol MN, Rajan VP, Zok FW. Compos. Pt. A Appl. Sci. Manufact. 2015, 74, 141-152.

[4] Yatim MYM, Shanmugam NE, Badaruzzaman WH. Thin Wall. Struct. 2015, 91, 13-28.

[5] Garcia D, Palazzetti R, Trendafilova I, Fiorini C, Zucchelli A. Compos. Struct. 2015, 130, 155-162.

[6] Aykul H. Sci. Eng. Compos. Mater. 2006, 13, 241-254.

[7] Loc TV, Jaehong L, Nguyen-Van H, Hung NX, Wahab MA. Int. J. Non-Linear Mech. 2015, 72, 42-52.

[8] Ansari R, Shojaei MF, Rouhi H, Hosseinzadeh M. Appl. Math. Model. 2015, 39, 2849-2860.

[9] Jun L, Hongxing H, Rongying S. Sci. Eng. Compos. Mater. 2008, 15, 285-302.

[10] Norouzi M, Rahmani H. Int. J. Ther. Sci. 2015, 94, 110-125.

[11] Tornabene F. Compos. Struct. 2011, 94, 186-206.

[12] Sofiyev AH. Compos. Struct. 2014, 113, 208-215.

[13] Zhang LW, Liew KM. Compos. Struct. 2016, 138, 40-51.

[14] Joodaky A, Joodaky I. Int. J. Mech. Sci. 2015, 100, 322-327.

[15] Tornabene F, Fantuzzi N, Viola E, Reddy JN. Compos. Pt. B Eng. 2014, 57, 269-296.

[16] Tornabene F, Ceruti A. World J. Mech. 2013, 3, 1-25.

[17] Lei ZX, Zhang LW, Liew KM. Appl. Math. Comput. 2015, 266, 773-791.

[18] Shariyat M, Hosseini SH. Compos. Struct. 2015, 124, 140-151.

[19] Almeyda YE, Ramos RR, Díaz RR, Castillero JB, Realpozo JCL, Montes HC, Sabina FJ, Lebon F. Int. J. Solids Struct. 2014, 51, 3508-3521.

[20] Chung DN, Dinh NN, Hui D, Duc ND, Trung NQ, Chipara M. J. Cur. Nanosci. 2013, 9, 14-20.

[21] Duc ND, Minh DK. Comput. Mater. Sci. 2010, 49, S194-S198.

[22] Hao S, Huang C, Zou B, Wang J, Liu H, Zhu H. Comput. Mater. Sci. 2012, 65, 254-263.

[23] Zhou C, Li K, Ma F. Comput. Struct. 2014, 139, 33-42.

[24] Deng X, Korobenko A, Yan J, Bazilevs Y. Comput. Methods Appl. Mech. Eng. 2015, 284, 349-372.

[25] Duc, ND, Thu PV. Compos. Struct. 2014, 109, 130-138.

[26] Duc ND, Quan TQ, Nam D. Mech. Compos. Mater. 2013, 49, 345-358. 
[27] Duc ND, Hadavinia H, Thu PV, Quan TQ. Compos. Struct. 2015, 131, 229-237.

[28] Vanin GA. Micro-mechanics of Composite Materials. Nauka Dumka, Kiev, 1985.
[29] Duc ND, Tung HV, Hang DT. Vietnam J. Mech. VAST 2007, 29, 58-64.

[30] Volmir AS. The Nonlinear Dynamics of Plates and Shells. Science Edition, Moscow, 1972. 